\title{
A NEW FULLY-AUTOMATIC PROCEDURE FOR THE IDENTIFICATION AND THE COUPLING OF THE OVERTOPPING WAVES
}

\author{
Sara Mizar Formentin ${ }^{1}$ and Barbara Zanuttigh ${ }^{1}$
}

\begin{abstract}
This contribution presents a new procedure for the automatic identification of the individual overtopping events. The procedure is based on a zero-down-crossing analysis of the water-surface-elevation signals and, based on two threshold values, can be applied to any structure crest level, i.e. to emerged, zero-freeboard, over-washed and submerged conditions. The results of the procedure are characterized by a level of accuracy comparable to the human-supervised analysis of the wave signals. The procedure includes a second algorithm for the coupling of the overtopping events registered at two consecutive gauges. This coupling algorithm offers a series of original applications of practical relevance, a.o. the possibility to estimate the wave celerities, i.e. the velocities of propagation of the single waves, which could be used as an approximation of the flow velocity in shallow water and broken flow conditions.
\end{abstract}

Keywords: wave overtopping events; wave identification; wave coupling; extreme overtopping volumes; wave celerities

\section{INTRODUCTION}

The prediction of the characteristics of the overtopping waves and their evolution over the dike crest and the identification of the individual overtopping volumes, are a key aspect in the design of coastal and harbor structures and for the estimation of their vulnerability (Van der Meer et al., 2012; EurOtop, 2016). Yet, the existing automatic procedures for the reconstruction of the individual overtopping volumes are affected by unaffordable inaccuracy in comparison with the manual or human-supervised analysis of the discharge time series (Hughes, 2015; Hughes \& Thornton, 2016). In addition, none of the existing procedures allows the coupling of the overtopping events propagating between two consecutive wave gauges.

In this contribution we are presenting the new and fully-automatic procedure recently developed by the authors (Formentin \& Zanuttigh, under review) for the identification and the coupling of the overtopping waves and volumes. The identification of the single overtopping events is based on a zerodown-crossing analysis of the wave signals and on two threshold values defined that are made varying with physical parameters, such as the wave height, the structure crest emergence or submergence, the structure roughness, etc. This new procedure includes a second algorithm for the coupling of the wave signals at two consecutive gauges, to estimate the velocity of propagation of the waves (celerity) and to capturing the evolution of the wave shape and parameters for an enhanced characterization of the overtopping process.

The whole procedure can be applied to measurements from both physical and numerical models or from prototype, for any structure emergence and submergence. It can process any kind of periodic signal in the time domain, including the discharge time series $\left(q,\left[\mathrm{~m}^{3} / \mathrm{s}\right.\right.$ per $\left.\left.\mathrm{m}\right]\right)$ for the individuation of the individual overtopping volumes $(\mathrm{V})$. In this case, the second step for the coupling of the signals is not necessary. The procedure has been verified against laboratory and numerical data, including smooth and rubble mound structures, emerged, zero-freeboard and submerged crest conditions. Such procedure is meant to represent a reliable and practical alternative to the manual techniques, and it will be provi ded for free upon request by e-mail to the first author.

The present contribution summarizes the main features and parameters characterizing the new procedure and illustrates its principal outcomes and applications of practical relevance. In particular, the identification algorithm is here applied to the reconstruction of the probability distribution of the extreme overtopping values and to the derivation of the values of the Weibull's function shape factors $b$. The resulting b-values are compared to existing b-values available from the literature for similar structure and to the predictions obtained by recent formulae (Hughes et al., 2012; Zanuttigh et al., 2013). An original application of the coupling algorithm, consisting in the extraction of the instantaneous and average vertical profiles of the wave celerities, is proposed.

The potentialities and the limits of the procedure, that are mainly related to the frequency of sampling characterizing the signals to be processed, are examined. The techniques to effectively check the consistency of the outcomes of the procedure are also discussed.

A few conclusions and recommendations are finally drawn. 


\section{OVERVIEW OF THE NEW PROCEDURE}

The new fully-automatic procedure includes two distinct algorithms: the wave identification and the wave coupling algorithm. The first algorithm can be applied to one or more $h$-signals (or $q$-signals) registered at one or more wgs and it is independent of the second one. It consists in the identification of the single overtopping events (or waves) from a zero-down-crossing analysis of the input signal. The wave coupling algorithm instead requires at least two input signals at two consecutive wgs, and recognizes the matching waves propagating from the first to the second wg. The coupling is based on the definition of the minimum and maximum time lags for the propagation of the waves between the two wgs. The scheme of Figure 1 gives a qualitative idea of the operating principle of the procedure and provides a reference for some of the symbols adopted in the following.

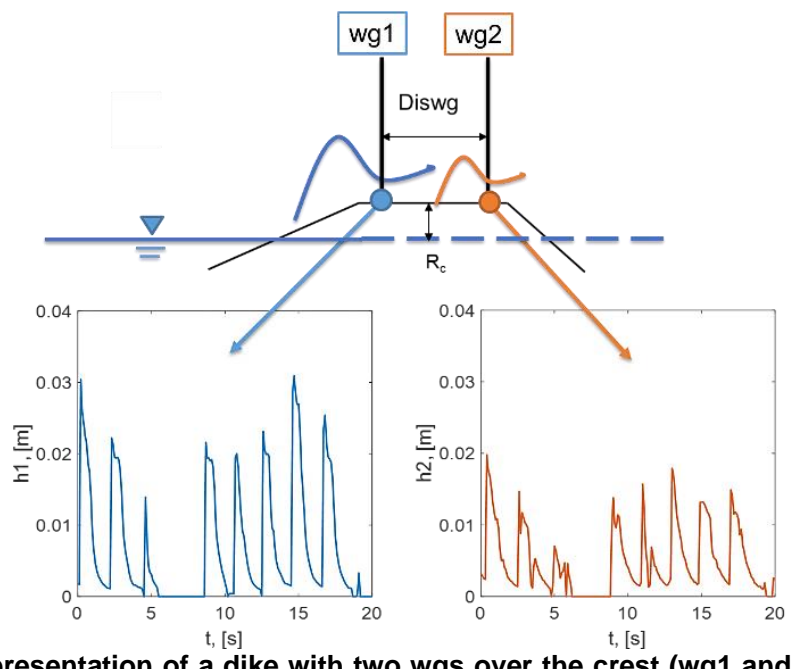

Figure 1. Schematic representation of a dike with two wgs over the crest (wg1 and wg2) and corresponding registrations of the $h$-signals ( $h 1$ and $h 2$ ) for the application of the wave identification and coupling procedure.

\section{Wave identification}

The wave identification algorithm can be applied to any kind of oscillatory signal in the time domain, such as, for example, the time series of the flow velocity $u$, of the wave overtopping discharge $q$, etc. For simplicity, the description of the algorithm, of its working principle and of its input and output parameters are made with reference to a sea surface elevation signal $h([\mathrm{~m}])$ registered at one resistant wave gauge (wg). Nevertheless, the explanation and the characterization of the parameters are meant to be of general validity.

The inputs of the wave identification algorithm are the time series of $h$-signals registered at one or more wgs (see Fig. 1) and the outputs are the time-ordered sequences of the specific wave overtopping events identified at each wg, see Fig. 2. The identification is based on a time-domain zero-down-crossing analysis (zdc, hereinafter) of the $h$-signal. Each overtopping event is identified when an element of the record $h$ equals a fixed threshold value representing the "zero" of the surface elevation signal. Such threshold can correspond to the still water level, to the mean of the $h$-signal, to 0 or to any value defined by the user. In the example of Fig. 1, this threshold " $l_{t h}$ " is represented by the filled-in circles and is set equal to the mean of the $h$-signal (which in this case is a positive value slightly greater than $0 \mathrm{~m}$ ).

The output time ordered sequences provided by the algorithm to characterize each identified event are:

- $\quad$ the instants of zero-down-crossing (Dc, [s]), i.e. the instant where $h=l_{t h}$;

- $\quad$ the crest and the trough wave heights (Zcr and Ztr, [m]);

- the instants of occurrence of the crest and trough wave heights (Icr and Itr, [s]).

The period of the i-th event is defined by the difference between two consecutive instants of zdc, $\operatorname{Dc}(\mathrm{i}-1)$ and Dc(i), while the height of the i-th event is defined by the difference Zcr(i)-Ztr(i). Five events and the corresponding Dc, Zcr, Ztr, Icr and Itr values are visible in Fig. 1. 


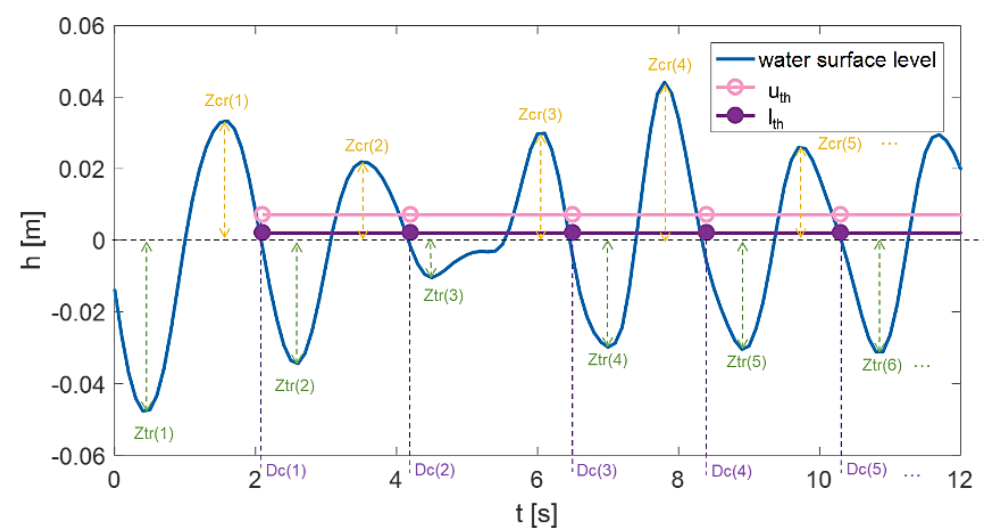

Figure 2. Example time series of a sea surface elevation signal $(h)$ with reference to the instants of zero-downcrossing (Dc), the crest $(\mathrm{Zcr})$ and the trough (Ztr) wave heights. The upper $\left(u_{t h}\right)$ and the lower $\left(I_{t h}\right)$ thresholds of the wave identification algorithm are marked with filled-in and empty circles, respectively.

The zdc procedure makes use of another threshold value, $u_{t h}$, which is represented in Figure 2 by void circles. The upper threshold $u_{t h}$ is set greater than a certain percentage of $l_{t h}$ and it is introduced in the algorithm to ensure a correct identification of the events also in case of very "irregular" or "noisy" wave signal, as in the example of Figure .3. This kind of signals typically occur over the crest of structures rarely overtopped, where the friction and/or the complete or partial wave breaking induce bursts of small amplitude and almost instantaneous duration in the sea surface elevation signal. These bursts are not waves and should not be identified by the algorithm as single overtopping events. The $u_{t h}$ is precisely used in the algorithm to discard all the oscillations of the $h$-signals whose amplitude is lower $\left(u_{t h}-l_{t h}\right)$. The burst around $132 \mathrm{~s}$ and all the small shape irregularities of the $h$-signal in the diagram of Fig. 3 are correctly not identified as waves because their amplitude is indeed lower $\left(u_{t h}-l_{t h}\right)$.

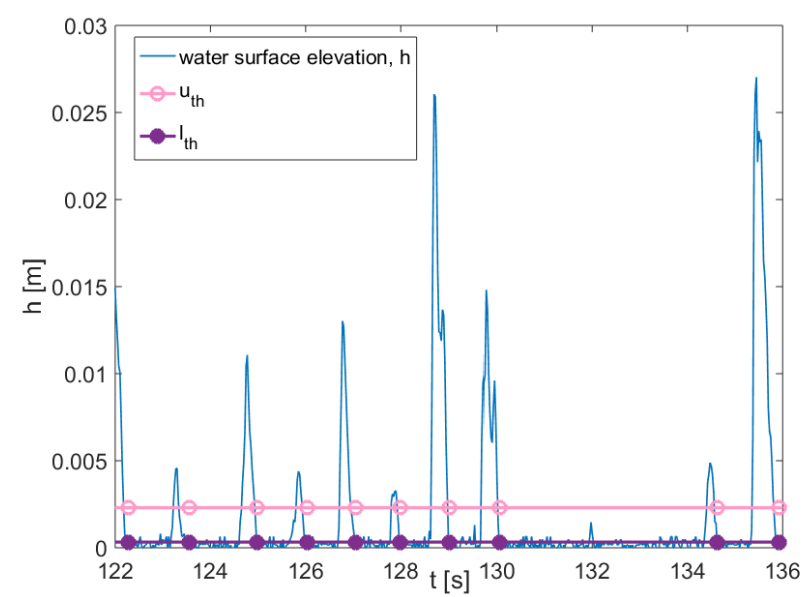

Figure 3. Example time series of a sea surface elevation signal $(h)$ registered over the crest of a rubble mound structure rarely overtopped. The signal presents significant shape irregularities and bursts due to the friction over the crest.

One of the most challenging aspects related to the wave identification is to give a "proper" determination of the $l_{t h}$ and $u_{t h}$. Overall, based on the experience collected by the authors (Formentin \& Zanuttigh, under review) on more than 200 numerical and experimental data including smooth and rubble mound structures, emerged, zero-freeboard, over-washed and submerged structures, the definition of $l_{t h}$ and $u_{t h}$ depends on:

- the structure crest freeboard $R_{c}$. The frequent or rare overtopping conditions characterizing the flow depth $h$ over the structure crest are firstly determined by the structure emergence or submergence. For submerged or over-washed structures $\left(R_{c} \leq 0\right)$, the oscillations of the $h$-signal are generally regular and larger (Fig. 2), while in emerged conditions $\left(R_{c}>0\right)$, the higher $R_{c}$, the lower the wave run-up, the lower the flow depth and the more irregular the wave shape (Fig. 3). Therefore, the difference $\left(u_{t h}-l_{t h}\right)$ should be lower for $R_{c}>0$ than for $R_{c} \leq 0$ to account for the smaller flow depths. 
- The significant wave height, $H_{s}$. The amplitude of the bursts of the $h$-signals to be discarded depends also on the amplitude of the incident waves: the higher $H_{s}$, the larger the oscillations and the bursts, and therefore the higher the difference $\left(u_{t h}-l_{t h}\right)$.

- The distance of the wg from the off-shore edge of the structure. The farther the wg, the greater the wave energy dissipation and the reduction of the amplitude of the wave at wg2 with respect to wg1.

- The structure roughness and permeability. The friction over the structure crest is enhanced in case of rough surfaces, resulting in enhanced wave shape irregularity. In case of permeable structures, the water percolates in the mound, determining a significant reduction of the flow depth amplitude and an increase of the bursts.

Of course, the definition of $l_{t h}$ and $u_{t h}$ depends also on the nature of the signal. If the wave identification algorithm is applied to a $q$-signal $u_{t h}$ and $l_{t h}$ should be a function of $q$ and it could be conservative to set $u_{t h}=l_{t h}$ in order to avoid the loss of overtopping volumes.

The default values of $u_{t h}$ and $l_{t h}$ in the procedure are set based on the following criteria:

- In case of an $h$-signal, $u_{t h}$ is increased by $\left[H_{s} / 10-H_{s} / 8\right]$ with respect to $l_{t h}$ in case of $R_{c} \leq 0$ and of $\left[H_{s} / 35-H_{s} / 10\right]$ in case of $R_{c}>0$. The entity of the increase varies between the two terms in brackets on the basis of the distance of the wg from the off-shore edge.

- In case of a $q$-signal, $u_{t h}$ is set equal to $q_{\text {mean }} / 10$, where $q_{\text {mean }}$ is the average value of $q$ over the time series, and $l_{t h}=0$.

These parameters can be customized by the user, who can decide to keep all the bursts also in case of an $h$-signal by simply setting $u_{t h}=l_{t h}$. Yet, the use of $u_{t h}>l_{t h}$ is recommended, especially if the wave coupling procedure is applied.

A qualitative hint of the level of the accuracy achieved by the new procedure in the identification of the overtopping volumes is given in Figure 4, showing the waves identified by the procedure applied to a time series of $q$ values for one of the tests by Hughes \& Thornton (2016). The new procedure gives the same results of Hughes \& Thornton (2016) without requiring any manual supervision. The accuracy of the procedure is basically determined by the values of $l_{t h}$ and $u_{t h}$, that in this case have been set respectively equal to $q_{\text {mean }} / 10$ and to he minimum between $q_{\text {mean }} / 4$ and $10^{-4} \mathrm{~m}^{3} / \mathrm{s}$ per $\mathrm{m}$, as in Hughes \& Thornton (2016). The full validation of the procedure is given in Formentin \& Zanuttigh (under review).

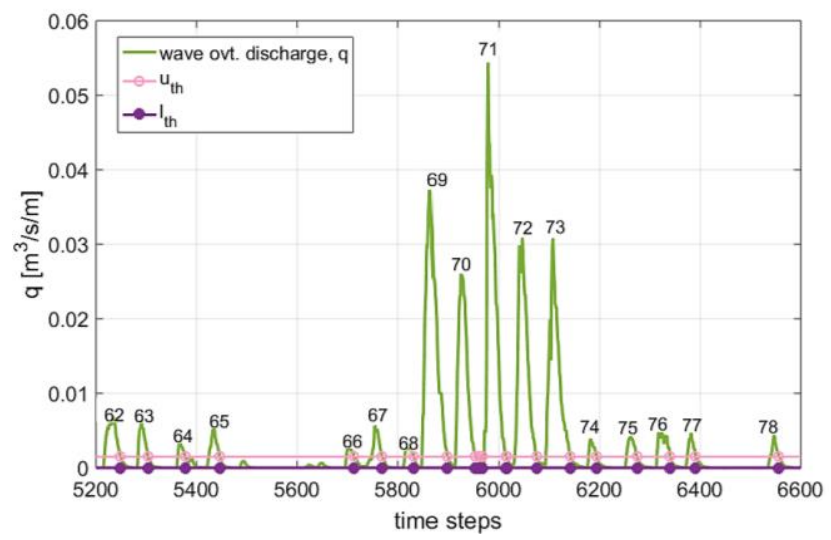

Figure 4. Time evolution of the overtopping discharge $(q)$ measured at the offshore edge of the dike crest for one of the tests by Hughes and Thornton (2016). The labelling of the waves is the same used by Hughes and Thornton (2016), see Fig. 1 in their work.

\section{Wave coupling}

The second algorithm of the procedure is dedicated to the coupling of the overtopping events. The application of this algorithm requires at least two signals registered at two consecutive wgs, namely wg1 and wg2 (see Fig. 1) and the outputs of the wave identification algorithm, i.e. the time ordered sequences of the overtopping events identified at wg1 and wg2 (Dc1 and Dc2, respectively). The wave coupling algorithm compares the sequences Dc1 and Dc2 and, computing the time lags necessary to the events to propagate from wg1 to $\mathrm{wg} 2$, recognizes the matching events, i.e. the same events that have been firstly identified at wg1 and then at wg2. The final outputs of the algorithm are the time ordered sequences of the "coupled events", Dc1s and Dc2s.

The two charts of Figure 5 provide an example of the $h$-signals registered at two consecutive wgs over the crest of an emerged rubble mound breakwater (experimental test from Kramer et al., 2005). In 
propagating between wg1 and wg2, the flow depth is subject to a non-negligible damping. In addition, the modification of the shape of the signal and the enhanced presence of bursts at wg2 lead to different numbers of waves identified at wg1 and wg2. The combination of these elements makes the wave coupling not straightforward, especially in case of rare overtopping and wave percolation in the permeable mound.
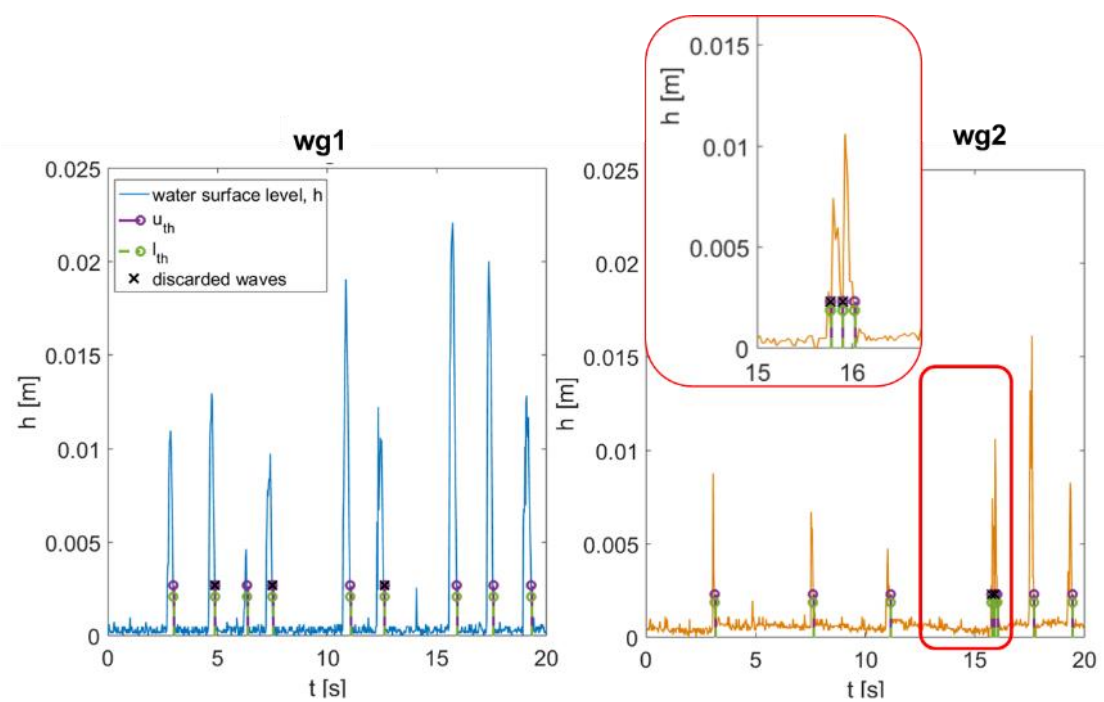

Figure 5. Example time series of the sea surface elevation signals $h 1$ and $h 2$ registered at two consecutive wgs placed over the crest of a rubble mound structure rarely overtopped.

The key element of the wave coupling algorithm is the definition of the minimum and maximum time lags, $d t_{\min }$ and $d t_{\max }$, necessary to the wave propagation. These values circumscribe indeed the possibility of an event identified at wg2 to be the same event "previously" registered at wg1. These time lags depend on:

- $\quad$ the distance between wg1 and wg2, diswg (see Fig. 1);

- the celerity $c$ of the single waves, which is proportional to the thickness of the water layer over the structure crest $h(c \propto \sqrt{g h})$. Therefore, $c$ - and $d t_{\min }$ and $d t_{\max }$ - vary also with the wave run-up, thus with the wave attack parameters $H_{s}$ and $T_{p}$ and with the structure freeboard $R_{c}$;

- $\quad$ the sample frequency of the signals, sf.

In the coupling algorithm, the following definitions of $d t_{\min }$ and $d t_{\max }$ have been set up:

$$
\left\{\begin{array}{l}
d t_{\text {min }}=\max \left(\frac{d i s w g}{c_{d w}} ; \frac{l}{s f}\right), \text { with } c_{d w}=\frac{L_{p}}{T_{p}} \\
d t_{\max }=\frac{d i s w g}{c_{s w}}, \text { with } c_{s w}=\min \left(\sqrt{g h_{l}}\right)
\end{array},\right.
$$

where $c_{d w}$ and $c_{s w}$ represent the celerity in deep water and in shallow water, respectively. $c_{d w}$ is assumed to be the theoretical maximum wave celerity determined by the deep water values of the peak wave length and period $L_{p}$ and $T_{p}$. The upper limit of $c$ determines in turn $d t_{\min }$. Yet, $d t_{\min }$ might be upperlimited by the minimum sampling time step, $1 / s f$ : if the adopted $s f$ is insufficient and the wave propagates from wg1 to $\mathrm{wg} 2$ with a $c$-value $>s f^{*} d i s w g$, the zdc analysis may register the $i$-th wave passage at the same time step, i.e. $\mathrm{Dc}_{1}(\mathrm{i})=\mathrm{Dc}_{2}(\mathrm{i})$. When this happens, the coupling procedure is forced to discard the event, resulting into a loss of data and an underestimation of the maximum and mean value of $c$. Further discussion about this topic is provided in the following sections of this contribution.

$c_{s w}$ is assumed to be the theoretical minimum wave celerity in shallow water, and it is estimated as the minimum of the values of the water surface elevation $h_{l}$ recorded at wg1 at the instants Icr $_{1}$. This value of $c_{s w}$ determines $d t_{\text {max }}$.

All the events that propagate from wg1 to wg2 with a time lag included in the interval $\left[d t_{\min } ; d t_{\max }\right]$ are coupled each other by the coupling algorithm. All the remaining events identified at wg1 or at wg2 that do not satisfy the coupling criteria based on the time lags are discarded from the wave coupling. In the example of Figure 5, the discarded events are marked with black crosses.

In summary, the coupling algorithm: 
- processes the outputs of the wave identification procedure, respectively $\mathrm{Zcr}_{1}, \mathrm{Ztr}_{1}, \mathrm{Dc} \mathrm{c}_{1}$ at $\mathrm{wg} 1$ and $\mathrm{Zcr}_{2}, \mathrm{Ztr}_{2}, \mathrm{Dc}_{2}$ at wg2;

- checks if the time of propagation of each event from wg1 to wg2 is included in the $\left[d t_{\min } ; d t_{\max }\right]$ and individuates the coupling events;

- discards all the events that cannot be coupled;

- provides as final output the time-ordered sequence of the "coupled events", i.e. $Z_{c r} r_{1 s}, Z_{t r} r_{1 s}, D_{1 s}$ and $\mathrm{Zcr}_{2 \mathrm{~s}}, \mathrm{Ztr}_{2 \mathrm{~s}}, \mathrm{Dc}_{2 \mathrm{~s}}$. The subscript "s" is used to refer to the "coupled events".

Note that the lengths (i.e. the number of the elements) of the records $\mathrm{Dc}_{1}$ and $\mathrm{Dc}_{2}$ may differ, while the lengths of $\mathrm{Dc}_{1 \mathrm{~s}}$ and $\mathrm{Dc}_{2 \mathrm{~s}}$ are equal, because each element of $\mathrm{Dc}_{1 \mathrm{~s}}$ does have a corresponding element in $\mathrm{Dc}_{2 s}$. For each couple of elements [Dc$\left.c_{1 s}(i), \mathrm{Dc}_{2 s}(\mathrm{i})\right]$, the difference $\mathrm{Dc}_{1 \mathrm{~s}}(\mathrm{i})-\mathrm{Dc}_{2 \mathrm{~s}}(\mathrm{i})$ represents the time of propagation of each single wave from wg1 to $\mathrm{wg} 2$.

An example of the results of the coupling procedure is given in Figure 6, which displays the coupled wave signals at $\mathrm{wg} 1$ and $\mathrm{wg} 2$ for a case of structure at negative freeboard. In this case the wave identification and the wave coupling are eased by the regularity of the signals, and only one identified at wg1 and uncoupled is discarded (event around $33 \mathrm{~s}$ marked with a black cross).

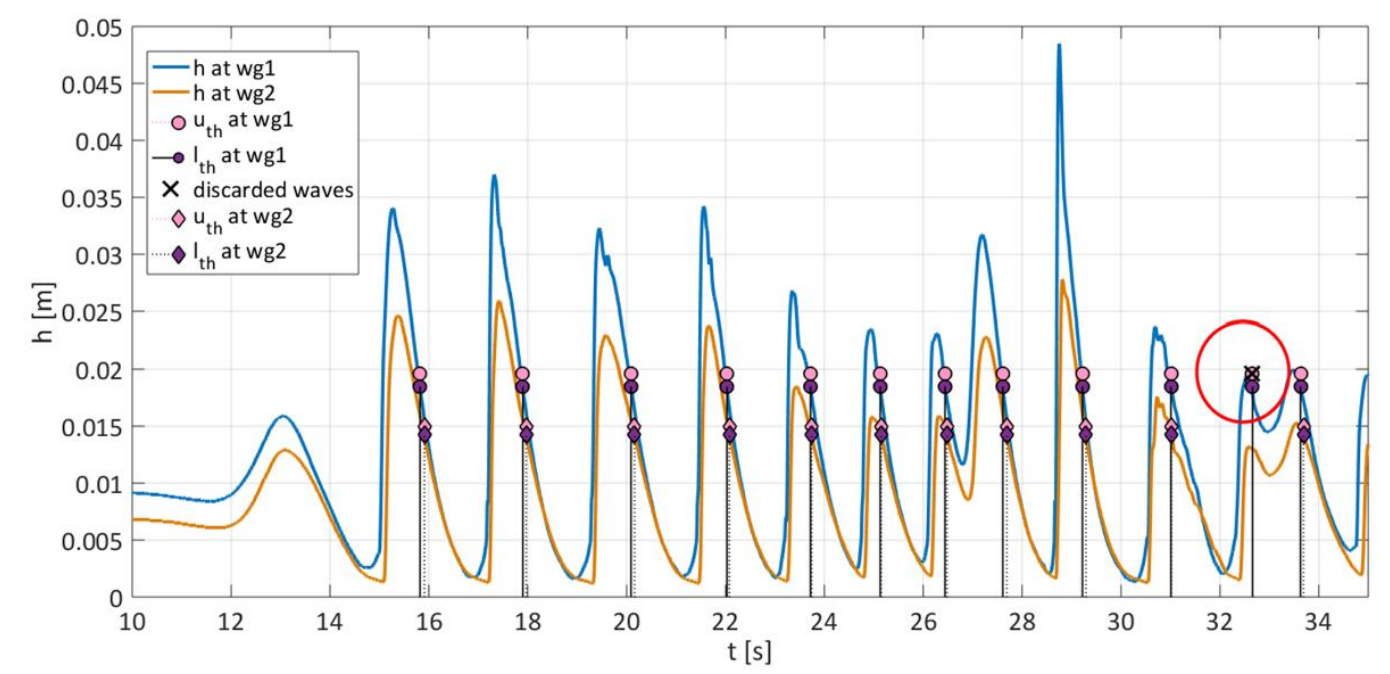

Figure 6. Example of the coupled time series of the sea surface elevation signals $h 1$ and $h 2$ registered at two consecutive wgs placed over the crest of a rubble mound structure frequently overtopped. The events that have been discarded by the procedure are marked with black crosses.

\section{Discussion}

The level of accuracy of the whole procedure is essentially determined by:

- the definition of the $u_{t h}$ and $l_{t h}$ values in the wave identification algorithm;

- the definition of $d t_{\min }$ and $d t_{\max }$ in the wave coupling algorithm;

- the sample frequency $s f$.

While the threshold values and the minimum and maximum time lags are subject to calibration and their adequacy can be checked and, if necessary, their values can be modified, the sample frequency represents an inherent constraint that cannot be changed and that can seriously affect the results of the procedure. As said above, a too low value of $s f$ with respect to diswg, can compromise the wave coupling, because the overtopping events may be identified at the same time step at the two wgs. On the contrary, an excessively high value of $s f$ may induce and/or enhance the noise of the wave signals, affecting the accuracy of the wave identification because it might become complicated to distinguish between "real" waves and bursts.

The sufficiency/insufficiency of $s f$ depends on several factors, such as: diswg, the actual celerity of the single waves, which depends in turn on $R_{c}$ and on the wave attack characteristics, etc. Therefore, a minimum fixed value of $s f$ cannot be indicated. As a general rule, it can be suggested to adopt, if possible, $s f \geq c_{d w} / d i s w g$. As for the maximum $s f$, it was found that the wave identification provided satisfactory results up to $s f=1000 \mathrm{~Hz}$ and that, therefore, the accuracy of the procedure is apparently less affected by the high frequencies.

Another relevant issue is the concrete possibility to properly check and assess the accuracy of the procedure. Indeed, the only way to rigorously validate the procedure would be the comparison of the 
number of the events automatically identified and coupled to the number events effectively recognized by human supervised analysis of the signals. However, this verification - which result extremely timeconsuming - would be still affected by a certain degree of subjectivity, because the identification of the overtopping events is left to the sensitivity of the human analysis, unless a fixed threshold value would be established a priori to accept or discard an event. Yet, the setting a fixed threshold value would essentially lead to an automatic detection.

A methodology to quantitatively assess the outcomes of the whole procedure is illustrated in the following.

The time-ordered sequences of the coupled events, $D c_{1 s}$ and $D c_{2 s}$, can be used to compute the timeordered sequences of the celerities $(c)$ of the single waves:

$$
c(i)=\frac{\operatorname{diswg}}{D c 2 s(i)-D c 1 s(i)}
$$

where $i$ is the $i$-th element of $\mathrm{Dc}_{1 \mathrm{~s}}$ and $\mathrm{Dc}_{2 \mathrm{~s}}$ (i.e. the $i$-th coupled event). For structures at zero or emerged crest freeboard $\left(R_{c} \geq 0\right)$, the $c$-values resulting from Eq. (2) can be compared with literature formulae for the estimate of the upper $2 \%$ values of the flow velocity $u, u_{2} \%$, at the dikes off-shore edge. The assumption that $c$ is approximately equal to $u$ is based on the observation that the flow over the crest of a dike at $R_{c} \geq 0$ typically occurs in shallow water and is characterized by broken waves only (Schüttrumpf \& Oumeraci, 2005). datasets:

To this purpose, the whole procedure has been applied to the data at $R_{c} \geq 0$ selected from the following

- 44 out of $942 \mathrm{D}$ numerical tests on wave overtopping against smooth dikes (off-shore slope $\cot \alpha_{\text {off }}=4$; $\left.6, R_{c} / H_{s}=[-1.5 ; 1.5]\right)$ collected by Formentin et al. (2014). The variety of the tested conditions includes: 44 tests in emerged or zero-freeboard conditions; 15 tests at negative freeboard and overwashed and overtopped conditions; 35 tests at negative freeboard and fully-submerged conditions. For all the tests, the dike crest width $G_{c}$ was kept constant and equal to $0.3 \mathrm{~m}$, and two wgs were placed at the off-shore edge and near to the in-shore edge, at the distance diswg $=0.27 \mathrm{~m}$. All the simulations were carried out at $s f=10 \mathrm{~Hz}$.

- 25 out of 33 3D tests against two permeable rubble mound breakwaters with a gap in between (offshore slope $\cot \alpha_{\text {off }}=2, R_{c} / H_{s}=[-1.5 ; 0.65]$ ) carried out in the shallow water basin of the Aalborg University by Kramer et al. (2005). The dataset included two structure configurations characterized by two crest widths $G_{c}$ and two corresponding diswg values: for the narrow crest width $\left(G_{c}=0.2 \mathrm{~m}\right)$, diswg $=0.15 \mathrm{~m}$, while for the wide crest width $\left(G_{c}=0.6 \mathrm{~m}\right)$, diswg $=0.4 \mathrm{~m}$. All the experiments on both the structure configurations were carried out at $s f=40 \mathrm{~Hz}$.

For each test, the distribution of the $c$-values for each identified and coupled overtopping event has been derived by means of Eq. (2). The upper $2 \%$ values of the distribution of the c-values, $c_{2} \%$, have been then extracted and compared to the most recent formulae for the prediction of $u_{2} \%$ at the structure offshore edge: Bosman et al. (2008) and Van der Meer et al. (2010). Both the formulae are based on the following expression:

$$
u_{2 \%}\left(x_{c}\right)=c_{u} \cdot\left[\left(R_{u, 2 \%}-R_{C}\right)\right]^{0.5}, R_{C} \geq 0
$$

where $R_{u, 2 \%}$ is the wave run-up exceeded by the upper $2 \%$ of the incoming waves to be computed following EurOtop (2016) and where $c_{u}=0.30 / \sin \left(\alpha_{\text {off }}\right)$ for Bosman et al. (2008) while $c_{u}=0.35 \cdot \cot \left(\alpha_{\text {off }}\right)$ for van der Meer et al. (2010). Both the formulae are targeted to structures at $R_{c} \geq 0$ and characterized by $\cot \alpha_{\text {off }}=[4 ; 6]$.

The $c_{2} \%$-values calculated with the new procedure are compared to the lines representing Eq. (3) in Figure 7. In this Figure, the data are plot as functions of the quantity $\left[g\left(R_{u, 2 \%}-R_{c}\right)\right]^{0.5}$ and are grouped by values of cot $\alpha_{\text {off. }}$. The $c_{2} \%$-values generally follow the trends of the formulae against $\left[g\left(R_{u, 2 \%}-R_{c}\right)\right]^{0.5}$ and most of the data are included between the lines. The effect of cot $\alpha_{\text {off }}$ is less remarked than as indicated by the formulae, because the data with $\cot \alpha_{\text {off }}=4$ and 6 are similarly distributed around the fitting lines and only a part of the experimental data, which are characterized by $\cot \alpha_{\mathrm{off}}=2$, are overestimated by the formulae, which are targeted for $\cot \alpha_{\text {off }}=4$ and 6.

One interest issue evident from Figure 7 is the effect of the $s f$ on the results of the procedure. Some of the $c_{2} \%$-values associated to the numerical data ( $\cot \alpha_{\mathrm{off}}=4$ and 6$)$ tend to be smaller than formulae predictions and, for all these data, the highest possible value of $c$ is $2.7 \mathrm{~m} / \mathrm{s}$, as imposed by diswg=0.27 
$\mathrm{m}$ and $s f=10 \mathrm{~Hz}$. These results indicate that the upper limit of $c$ imposed by $s f$ affects the whole distribution of the $c$-values, resulting in a non-conservative estimation of all the $c_{2} \%$ values.

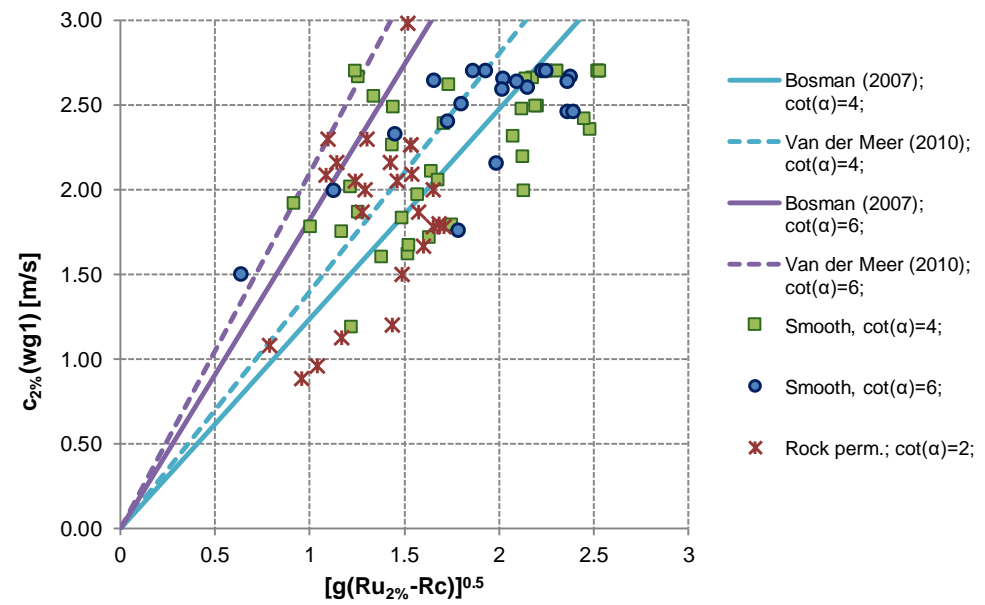

Figure 7. Comparison among literature formulae (Bosman et al., 2008; van der Meer et al., 2010) and values of the c2\% computed with the new procedure for the data on smooth dikes by Formentin et al., 2014 (cota=4;6, squares and circles, respectively) and on rock permeable structures by Kramer et al., 2005 (cota=2, stars).

\section{ANALYSIS OF THE EXTREME OVERTOPPING VOLUMES}

A direct application of the wave identification procedure is the reconstruction of the distribution of the individual overtopping volumes $V\left[\mathrm{~m}^{3} / \mathrm{m}\right]$ of a structure. To illustrate this application, the wave identification algorithm of the new procedure has been applied to the numerical time series of the wave overtopping discharge $q\left[\mathrm{~m}^{3} /(\mathrm{s} \cdot \mathrm{m})\right]$ available for the 94 numerical tests by Formentin et al. (2014) (see above).

These $q$-time series have been reconstructed by integrating the time series of the on-shore directed flow velocities $(u)$ with the corresponding flow depth values $(h)$ at the off-shore edge of the dike crest prompted by the numerical simulations.

As shown in Figure 4 for the test by Hughes \& Thornton (2016), the procedure identifies the overtopping events from the elaboration of the $q$-signal by setting the values of $u_{t h}$ and $l_{t h}$ as function of $q$, and specifically: $u_{t h}=\operatorname{mean}(q) / 10$ and $l_{t h}=0 \mathrm{~m}^{3} /(\mathrm{s} \cdot \mathrm{m})$.

For each identified overtopping event, the corresponding volume $V$ is calculated through the numerical integration of the $q$-signal between the two consecutive instants of zdc, Dc(i-1) and Dc(i). The example of Figure 8 graphically represents the volumes resulting from the integration of the $q$-signal once all the individual overtopping events are identified.

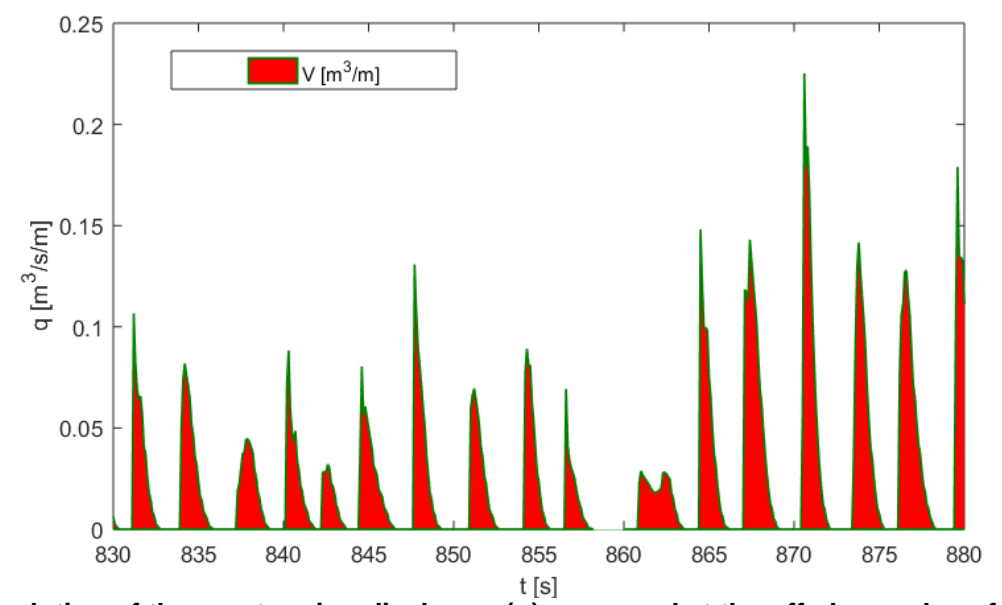

Figure 8. Time evolution of the overtopping discharge $(q)$ measured at the off-shore edge of the dike crest for one of the numerical tests by Formentin et al. (2014) and corresponding volumes (V) derived from the integration of $q$. 
According to the literature (inter alia, EurOtop, 2016), the probability exceedance distribution of the $V$-values can be approximated by a Weibull function:

$$
P\left(V_{i} \geq \bar{V}\right)=\exp \left(-\left(\frac{V}{a}\right)^{b}\right)
$$

where $P\left(V_{i} \geq \bar{V}\right)$ (or simply $P$ ) is the probability that the $i$-th individual volume $V_{i}$ is greater than a specified volume $\bar{V}$. The parameters $a$ and $b$ are the Weibull distribution scale and shape factors, respectively. Generally, the literature formulae parametrize $a$ as the mean or a function of the mean of the distribution of the $V$-values (Van der Meer and Janssen, 1994, updated by Van der Meer et al., 2010; Victor et al., 2012).

The charts of Figure 9 show an example of the exceedance probability distribution of $V$ derived with the new procedure and the explained methodology for a test at zero-freeboard of the selected database. In this Figure, the $V$-values (abscissa) are normalized with the mean of the distribution $\left(V_{b a r}\right)$ and the data are shown in the double logarithmic scale to fit the Weibull trends (straight lines). The angular coefficients of the Weibull fits are the shape $b$ factors.
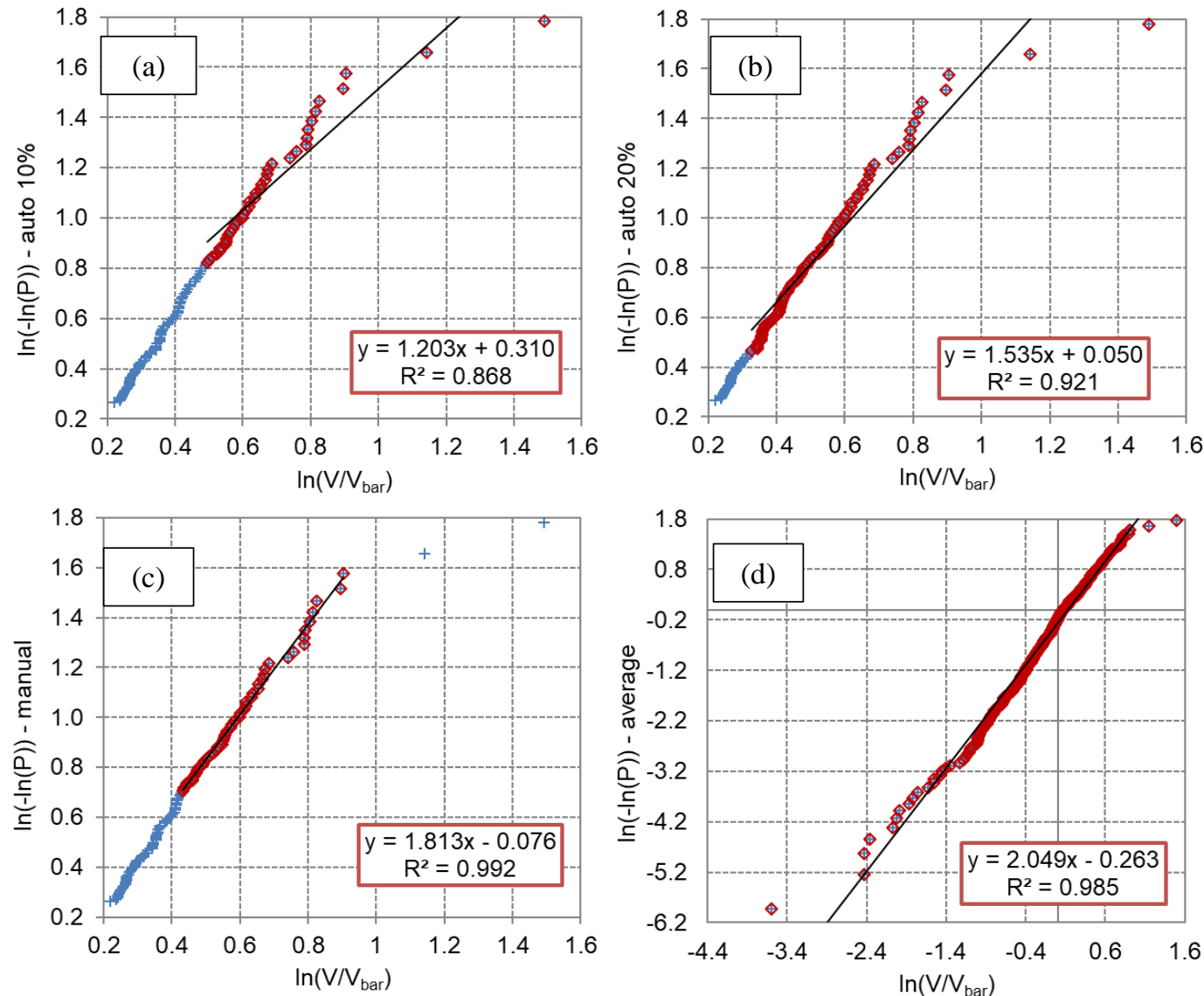

Figure 9. Example of fittings of the Weibull distribution of the individual wave overtopping volumes $(V)$ derived from a numerical simulation. The 4 different fittings are obtained from an automatic selection of the upper $10 \%$ and $20 \%$ volumes (panels a and b, respectively), from a manual selection of the extreme volumes (panel c) and from the whole distribution of the volumes (panel d).

In the literature, various methods to determine the $b$-values are proposed. Victor et al. (2012) fitted the Weibull distribution on the upper $50 \%$ of the volumes, while Hughes et al. (2012) demonstrated that the fitting on the upper 10\% gave a better representation of the highest values. In Zanuttigh et al. (2013), it is suggested to adopt a manual identification including approximately the $20 \%$ highest values.

Since we are interested in the extreme wave overtopping volumes, the $b$-values are based on the Weibull fit of the "upper tail" of the distributions (i.e. $\leq 20 \%$ ). 
Figure 9 compares the $b$-values obtained by fitting the Weibull on 4 different portions of the $V$ distribution:

- the value of $b=2.049$ (panel d) corresponds to the average fit of all the $V$-values and it is included for comparison;

- the values $b=1.203$ and $b=1.535$ (panels a and $\mathrm{b}$ ) are derived from the automatic fitting of the highest $10 \%$ and $20 \% \mathrm{~V}$-values, respectively;

- $\quad b=1.813$ (panel c) is determined by manually fitting the upper tail of the $V$-distribution.

The manual identification is verified by checking the sensitivity of the values of $R^{2}$ to the amount of data selected for the linear fitting. The upper $20 \%$ values are included as starting guess and some "outliers" are discarded. In the example of Figure 9, the highest 2 volumes of the distribution, that are clearly aligned on a milder line, have been considered "outliers". The total number of overtopping volumes is 379 in this case, and the manually-identified volumes are 57.

Two expressions are here considered for the shape factor $b$.

- The formula by Hughes et al. (2012), that updates the formulation given by Victor et al. (2012), and expresses $b$ as a function of the relative crest-freeboard $R_{c} / H_{s}$ :

$$
b=\left(\exp \left(-0.6 \frac{R_{c}}{H_{s}}\right)\right)^{1.8}+0.64
$$

Eq. (5) was calibrated on the basis of a variety of experimental tests (Victor et al., 2012; Hughes and Nadal, 2009; Van der Meer and Janssen, 1995) on smooth dikes in emerged and submerged conditions and characterized by various slopes.

- Zanuttigh et al. (2013) presented the combined analysis of the Weibull $b$-value for low-crested rubble mound structures, smooth slopes and conventional rubble mound breakwaters, resulting in the following new trend for the $b$-value:

$$
b=0.73+55 \cdot\left(\frac{q}{g H_{s} T_{m-1,0}}\right),
$$

Another formulation by Pan et al. (2015) is also available, but it is not used in this work as it applies to submerged cases only.

It is worthy to remind that Eq.s (5) and (6) have been derived from fitting respectively, the upper $10 \% V$-values, and the manually-identified upper part (approximately the 20\%) of the $V$-distribution.

The $b$-values calculated for all the numerical simulations are compared to the Eq.s (5) and (6) and to the $b$-values obtained for similar data on smooth structures by Hughes \& Nadal (2009) and by Victor, et al. (2012) in Figure 10. For each formula, the $b$-values determined on the basis of the automatic $(20 \%$ and $10 \%$ upper values) or the manual criterion are shown on three different plots. The manual identification gives the best agreement among the $b$-values and the formulae (panels a and $\mathrm{b}$ ). As expected, a similar result is obtained with the fixed $20 \%$ threshold (panels c and d), while more scatter is observed when the $10 \%$ is used (panels e and $\mathrm{f}$ ).

In the charts of Figure 10, the numerical data are distinguished among "submerged" and "overwashed/overtopped" structures because Eq. (5) is targeted to over-washed/overtopped conditions only. Actually, for a given value of $R_{d} / H_{s}$, the submerged conditions give significantly lower values of $q$ if compared to the over-washed/overtopped conditions, because the presence of the water at the landward slope reduces the hydraulic gradient between the off-shore and the in-shore edge of the structure crest, nullifying the overflow contribution of the overtopping (see Formentin et al., 2014). According to Eq. (6), the lower $q$, the lower $b$ : therefore, it expected that, for the same values of $R_{c} / H_{s}$, the submerged tests provide lower values of $b$ than Eq. (5). Indeed, when $R_{c} / H_{s}<0$, the numerical $b$-values associated to the submerged structures (blue circles) follow a milder trend with respect to the over-washed structures (red stars), the other experimental data and the curve of Eq. (5). On the contrary, both the overwashed/overtopped and the submerged tests follow the same trend with $q / g H_{s} T_{m-1,0}$ (panels b, d, f) showing a good agreement with Eq. (6).

It can be concluded that the shape factor $b$ is strongly dependent on $q$. The values of $\mathrm{b}$ obtained with the new procedure are accurately fit by Eq. (6), suggesting that the wave identification procedure can be successfully adopted for the reconstruction of the distribution of the wave overtopping volumes. 

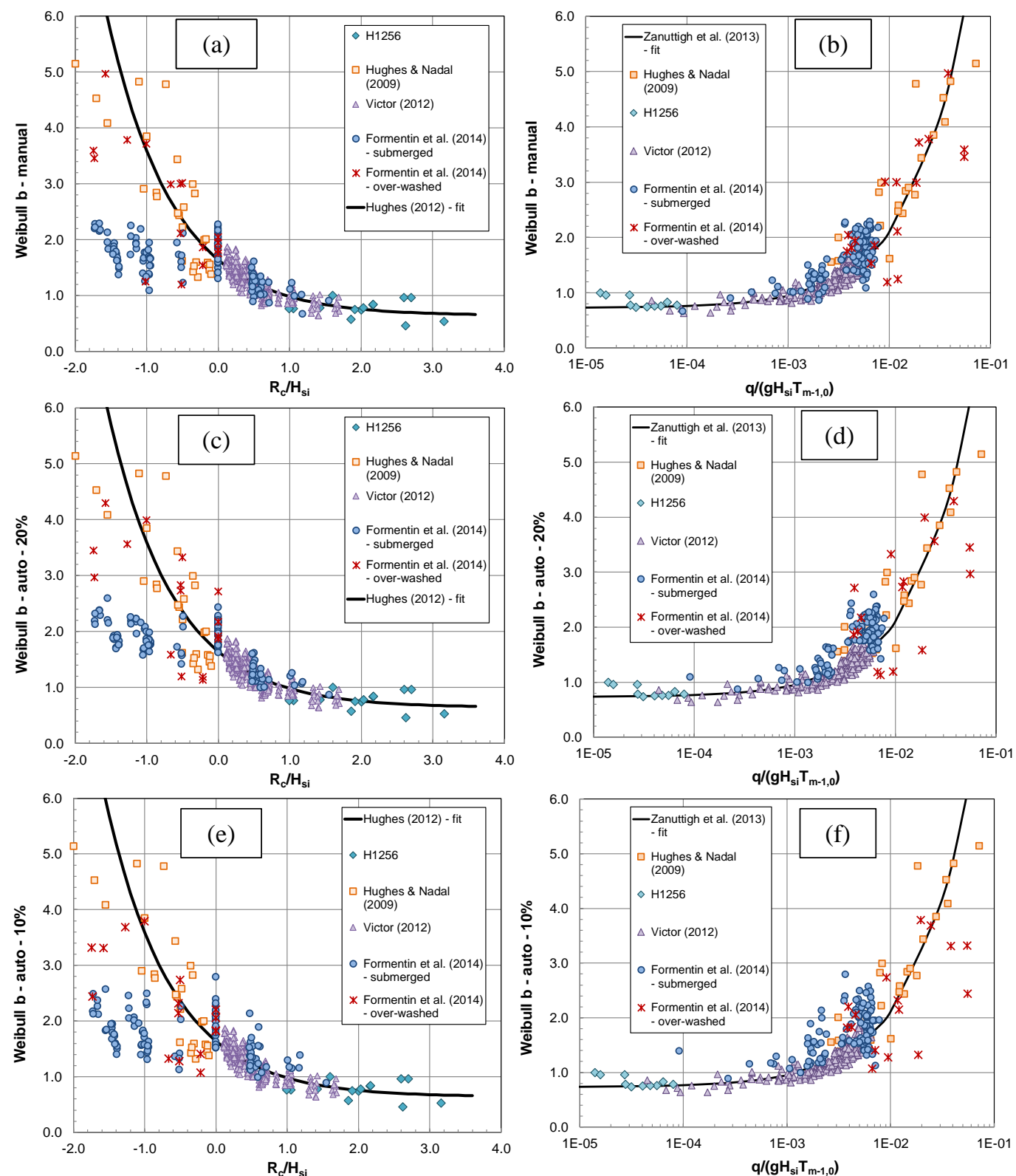

Figure 10. Values of $b$ derived from the numerical simulations and relationships for smooth structures. Panels a, c, e: Eq. (5) by Hughes et al., 2012; panels b, d, f: Eq. (6) by Zanuttigh et al., 2013. Panels a,b: $b$-values manually determined; panels c, d: fitted on the $20 \%$ upper volumes; panels $e, f$ : fitted on the $10 \%$ upper volumes.

\section{WAVE CELERITIES}

As already shown, the wave celerities $c$ may also represent a valid approximation of the wave velocity $u$ in conditions far from the wave breaking. Especially in case of physical modelling, the computation of $u$ may be expensive and not straight-forward (Blenkinsopp et al., 2010). Indeed the measurements of the velocities in breaking conditions is rather difficult, requiring in many cases the setup of an ad-hoc equipment as for the floaters used in the Wave Overtopping simulator (van der Meer et al., 2012). The use of Acoustic Doppler Profilers is a good option only for limited layer thicknesses, while the use of the PIV is rather expensive and complicated and may be affected by the foam. In most cases, the velocity is measured in the lab and in the prototype with Acoustic Doppler Velocimeters, leading to punctual measures associated to the fixed position of the velocimeter.

The new procedure allows instead to evaluate the average values of $c$ but also the local instantaneous values along each (coupled) wave and along the vertical profile of each wave. The vertical profiles of $c$ for each couple of waves can be derived by re-applying the wave identification and the wave coupling 
algorithms several (n) times, and by modifying each time the values of $u_{t h}$ and $l_{t h}$. For each pair of $\left(u_{t h}\right.$; $\left.l_{t h}\right)$ values, i.e. for each re-application of the procedure, the time ordered sequences of the instants of z.d.c. of the coupled waves, $\mathrm{Dc}_{1 \mathrm{~s}}$ and $\mathrm{Dc}_{2 \mathrm{~s}}$, and the corresponding wave celerities computed on the basis of Eq. (2) are thus associated to different elevations of the $h$-signals.

An example is given in Figure 11, which reports the results of this application to one of the tests on permeable structures at $R_{c}=0$ from the database by Kramer et al. (2005). Figures $11-\mathrm{a}$,b show the first 30 seconds of the time series of the signals $h 1$ and $h 2$ (panels a and b), displaying the $\mathrm{n}=10$ values of $u_{t h}$ used for the $\mathrm{n}=10$ re-applications of the procedure (circles and diamonds for $h 1$ and $h 2$, respectively). The corresponding vertical profile of the average celerities, $c_{\text {mean }}$, calculated in correspondence of 10 values of $u_{t h}$ are displayed in Figure 11-c. Figure 12-c provides instead the instantaneous vertical profile of $c$ derived for one of the overtopping events (Figure 12-a,b) identified and coupled by the procedure (specifically, the wave \#8 of the time series $h 1$ and $h 2$ of Figure 11).

In both Figures 11-c and 12-c, a significant variability of $c$ (up to the $30 \%$ ) is detected between the wave trough and the wave crest. As expected, considering both the instantaneous and the average profiles, $c$ tends to increase from the trough to the crest, where it reaches its maximum value.
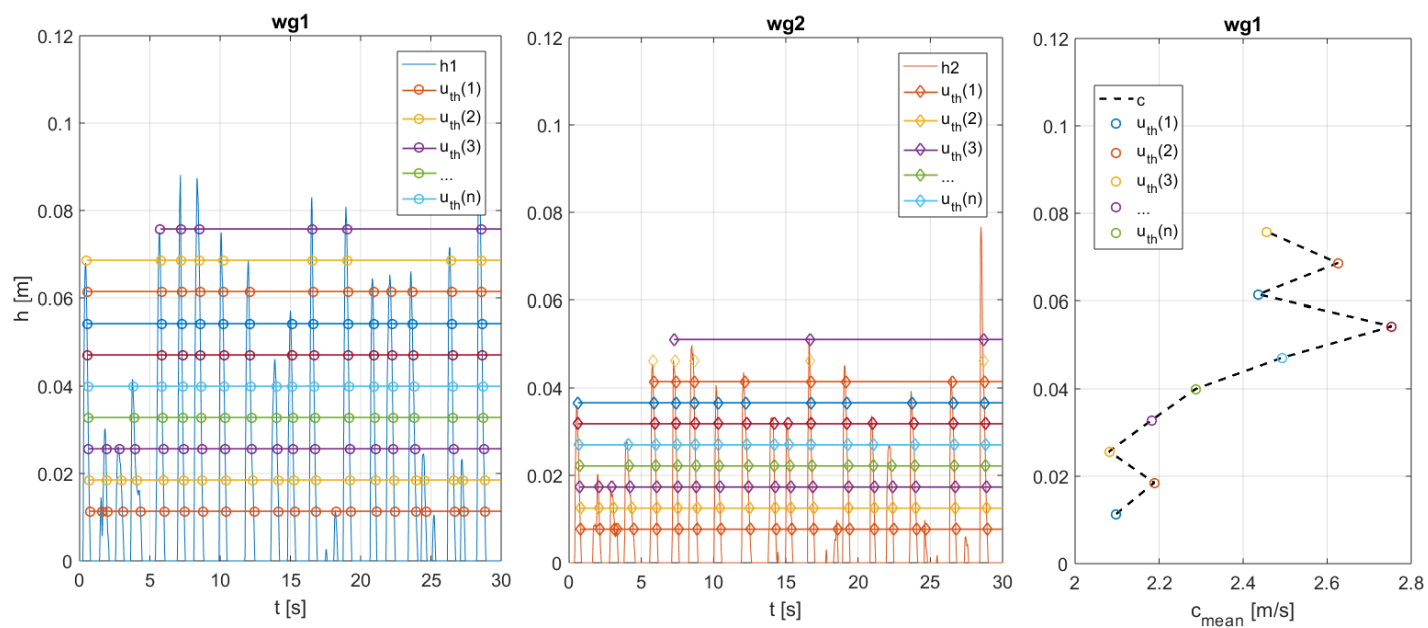

Figure 11. Example time series of the sea surface elevation signals $h 1$ and $h 2$ registered at two consecutive wgs (wg1 and wg2) placed over the crest of a rubble mound structure (panels a and b) and vertical profile of the mean wave celerity $c$ (panel c) computed by repeating the new procedure several times with different $u_{t h}$ values (circles in the panel $a$ and diamonds in the panels $b$ and $c$ ).
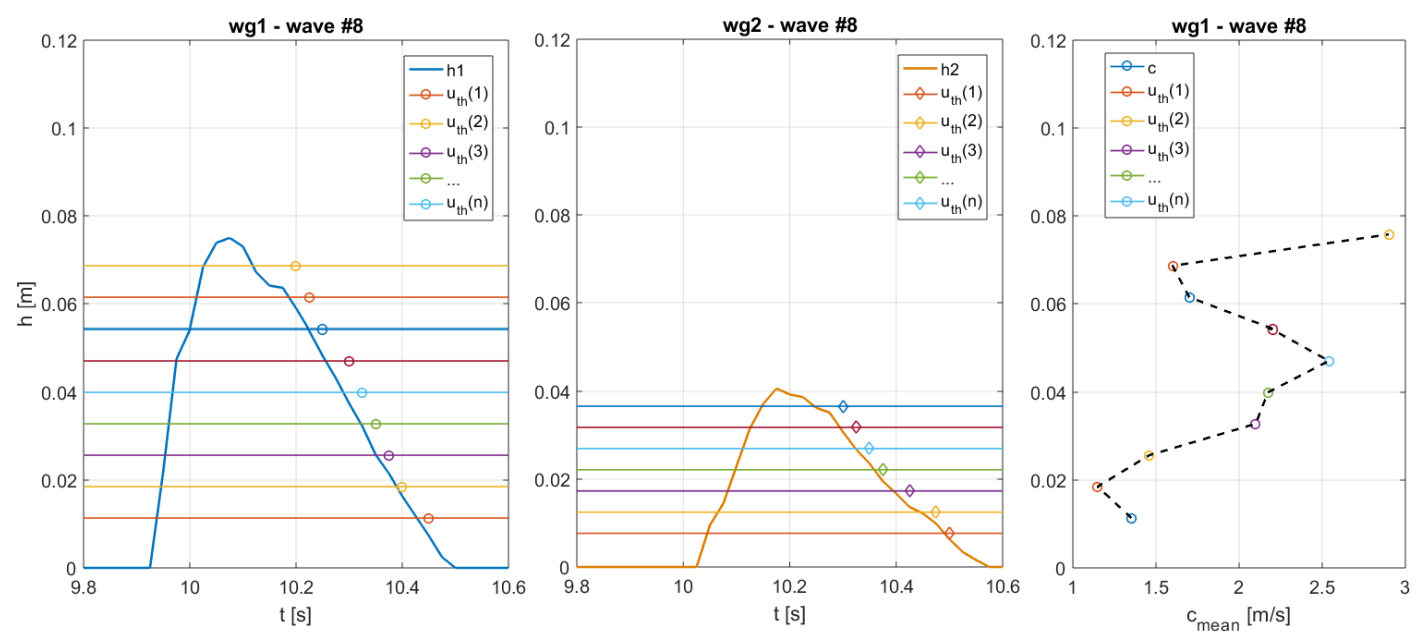

Figure 12. Panels a and b: example of a coupled wave (\#8) at wg1 and wg2, with indication of the points where the wave celerity has been extracted. Panel c: vertical profile of the corresponding wave celerities $c$. 


\section{CONCLUSIONS}

This paper has presented the new procedure for the identification and the coupling of the single wave overtopping events recently developed by the authors (Forment \& Zanuttigh, under review). The procedure is fully automatic, i.e. does not require the human supervision. It is organized into two sequential algorithms, the wave identification and the wave coupling. The first algorithm identifies the single waves based on a zero-down-crossing analysis of the water level signal and on two level thresholds values. The identification step of the procedure has been applied in this paper to the analysis of the extreme overtopping volumes for a dataset of numerical tests on smooth dikes (Formentin et al. 2104). For each test, the statistical distribution of the volumes and the corresponding Weibull's shape factors $b$ have been reconstructed by applying the wave identification algorithm to the processing of the wave overtopping discharge time series. The resulting $b$-values follow the trends of the predicting formulae (Hughes et al., 2012; Zanuttigh et al., 2013) and are consistent with other existing $b$-values for similar smooth structures available from the literature (Hughes \& Nadal, 2009; Victor et al., 2012).

The second algorithm couples the waves at two consecutive gauges, computing the time lag necessary for the wave propagation of the wave event based on the definition of the minimum and maximum possible time lags. The coupling step allows also the calculation of the wave celerity, which can be used in turn as estimator of the flow velocity in shallow water conditions and to get thus estimates of the wave overtopping discharge.

The coupling procedure is applied to a set of experiments on permeable structures (Kramer et al., 2005 ) to derive the instantaneous and average vertical profiles of the wave celerities. The results of the application indicate that the $c$-values tend to increase from the wave trough to the wave crest, with an average variability of the $30 \%$ along the vertical profile.

The accuracy of the whole procedure is comparable to the accuracy of a manual analysis of the wave signals. It has been shown in this contribution that the main limit of the procedure is related to the sample frequency of the input wave signals, that represents an inherent constraint independent of the parameters of the procedure itself.

\section{ACKNOWLEDGEMENTS}

The support of the European Union's Horizon 2020 research and innovation program under grant agreement 700699 BRIGAID ("BRIdging the GAp for Innovations in Disaster resilience") is gratefully acknowledged.

\section{REFERENCES}

Blenkinsopp, C.E., Turner, I.L., Masselink, G. and Russell, P.E., 2010. Validation of volume continuity method for estimation of cross-shore swash flow velocity, Coastal Engineering 57(10), pp. 953-958.

Bosman, G, J.W. Van der Meer, G. Hoffmans, H. Schüttrumpf and Verhagen., H.J., 2008. Individual overtopping events at dikes. ASCE, proc. ICCE 2008, Hamburg, Germany, p. 2944-2956.

Eurotop. 2016. In: Pullen, T., Allsop, N.W.H., Bruce, T., Kortenhaus, A., Schüttrumpf, H., van der Meer, J.W. (Eds.), Wave Overtopping of Sea Defences and Related Structures - Assessment Manual. www.overtopping-manual.com.

Formentin, S.M. and Zanuttigh, B. A fully-automatic procedure for the identification and coupling of individual overtopping waves, under review.

Formentin, S.M., Zanuttigh, B., van der Meer, J.W. and Lara, J.L., 2014. Overtopping flow characteristics at emerged and over-washed dikes, Proc. of XXXIV ICCE, Seoul (ROK).

Kramer, M., Zanuttigh, B., van der Meer J. W., Vidal, C. and Gironella, X., 2005. Laboratory experiments on low-crested structures, Coastal Engineering, 52, 867-88.Hughes, S.A., Thornton, C.I., van der Meer, J.W. and Scholl, B., 2012. Improvements in describing wave overtopping processes. ASCE, Proc. ICCE 2012, Santander, Spain.

Hughes, S.A. (2015). Hydraulic parameters of overtopping wave volumes, Proceedings of Coastal Structures and Solutions to Coastal Disasters 2015, Boston, MA.

Hughes, S. A., and Nadal, N. C. Laboratory study of combined wave overtopping and storm surge overflow of a levee, Coastal Engineering, Elsevier, 56(3), 2009, pp.244-259.

Hughes, S.A., Thornton, C.I., van der Meer, J.W. and Scholl, B., 2012. Improvements in describing wave overtopping processes. ASCE, Proc. of XXXIII ICCE, Santander, Spain.

Hughes, S.A. and Thornton, C.I. (2016). Estimation of time-varying discharge and cumulative volume in individual overtopping waves, Coastal Engineering, 117, 191-204. 
Schüttrumpf H., and Oumeraci, H. 2005. Layer thicknesses and velocities of wave overtopping flow at sea dikes, Coastal Engineering, 52 (6), 473-495.

Van der Meer, J.W. and Janssen J.P.F.M., 1994. Wave run-up and wave overtopping at dikes and revetments, Delft Hydraulics.

Van der Meer, J.W., B. Hardeman, G.J. Steendam, H. Schüttrumpf and H. Verheij, 2010. Flow depths and velocities at crest and inner slope of a dike, in theory and with the Wave Overtopping Simulator. ASCE, Proc. XXXII ICCE, Shanghai, China.

Van der Meer, J.W., Provoost, Y and Steendam, G.J., 2012. The wave run-up simulator, theory and first pilot test. ASCE, Proc. ICCE 2012, Santander, S.

Victor, L., 2012. Optimization of the Hydrodynamic Performance of Overtopping Wave Energy Converters: Experimental Study of Optimal Geometry and Probability Distribution of Overtopping Volumes. Phd, UGent, Zwijnaarde; Belgium.

Zanuttigh, B., van der Meer, J.W., Bruce, T. and Hughes, S. (2013). Statistical characterisation of extreme overtopping wave volumes. Proc ICE, Coasts, Marine Structures and Breakwaters 2013, Edinburgh, UK. 